\title{
1. Double-layered Water Cherenkov Detector for SWGO
}

2 Samridha Kunwar ${ }^{a, *}$ on behalf of the SWGO Collaboration

3 (a complete list of authors can be found at the end of the proceedings)

$4 \quad{ }^{a}$ Max-Planck-Institut für Kernphysik (MPIK), Saupfercheckweg 1, 69117 Heidelberg, Germany

$5 \quad$ E-mail: samridha.kunwar@mpi-hd.mpg.de

The Southern Wide-field Gamma-ray Observatory (SWGO) will use the well-established and cost-effective technique of detecting Cherenkov light produced in water-filled detection units for $\mathrm{TeV}$ gamma-ray astronomy. Leveraging detector material reflectivity together with an optimised aspect ratio is an option to improve the performance of an array of such detector units. The double-layered Water Cherenkov Detector units comprise chambers with single photosensors in each. A reflective upper compartment enhances sensitivity to impinging secondary particles. A shallow lower compartment enables muon tagging and consequently improves the gamma hadron separation power of the observatory. Here we present detailed studies on the double-layered unit design.

$37^{\text {th }}$ International Cosmic Ray Conference (ICRC 2021)

July 12 th - 23rd, 2021

Online - Berlin, Germany

\footnotetext{
*Presenter
} 


\section{Introduction}

The induced electromagnetic cascade produced by air showers are well suited to be observed by ground-level particle detectors providing intrinsically wide field-of-view and $\sim 100 \%$ duty cycle. HAWC ( High-Altitude Water Cherenkov) [1] on the flanks of the Sierra Negra in Mexico, and LHAASO (Large High Altitude Air Shower Observatory) [2] in the eastern Tibetan plateau are the two main instruments currently under operation that comprise an array of Water Cerenkov Detector (WCD) units. The detection of $\gamma$-rays higher than $0.1 \mathrm{PeV}$ have already been reported by LHAASO [3] and instrumenting the Southern Hemisphere will provide unprecedented opportunities to probe the galactic plane and the southern hemisphere further [4].

Furthermore, instrumenting at a High altitude $(>4.4 \mathrm{~km})$ with a high fill factor $(>80 \%)$ will allow SWGO to be complementary in the same energy range as Imaging Atmospheric Cherenkov Telescopes (IACTs). This is extended to an outer array with a fill factor of $8 \%$ as in Fig. 1 that aims to improve sensitivity at higher energies.

SWGO (for an overview on status and prospects, see [5]) is investigating several detector technologies such as units with multiple photo-sensors [6] and an option to deploy detector units in a lake [7]. Muon identification with a separate detector element is a reasonable means of hadronic background rejection (see, e.g. [8]) for $\gamma$-ray astronomy. Here we develop the concept of a double-layered WCD design; as a potential detector unit for SWGO, comprising two isolated chambers where the lower chamber in conjunction with the upper chamber enables an effective method for gamma/hadron separation. The detector unit with optimised aspect ratio and material reflectivity will also have improved particle detection efficiency and angular resolution.

The simulations in this work use

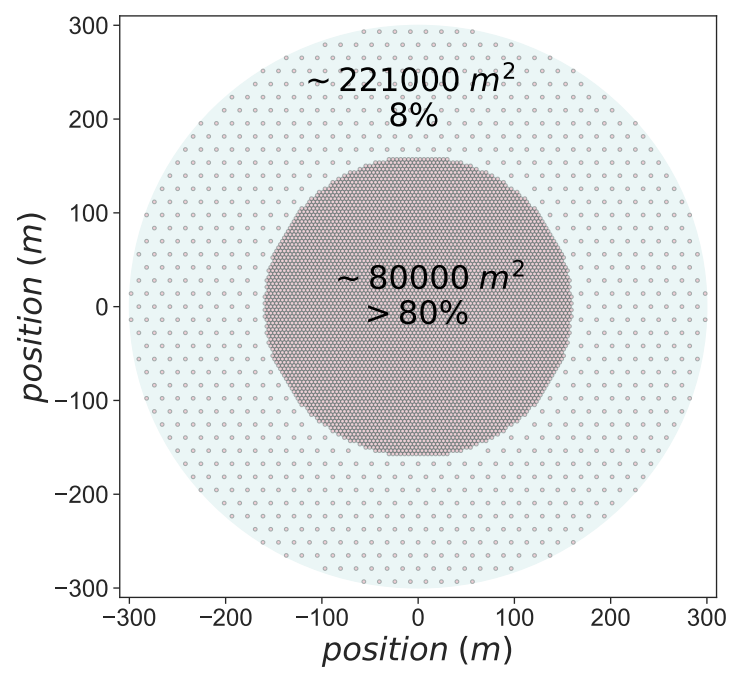
GEANT4 [9] within a simulation framework adapted from the HAWC collaboration. Air Shower simulations use the CORSIKA 7.7400 simulation package [10] where we select the hadronic interaction model QGSJet-II.04 [11] for energies above $80 \mathrm{GeV}$. UrQMD 1.3.1 $[12,13]$ treats the low energy hadronic interactions and for electromagnetic processes, we use the EGS4 electromagnetic model [14].

\section{Unit Design}

The double-layered design comprises two chambers that are isolated from each other, as shown in Fig. 2. The upper chamber is a light-tight chamber with a reflective lining and a centrally located 8" Photo-Multiplier Tube (PMT) facing upwards. The PMT orientation ensures that the prompt 


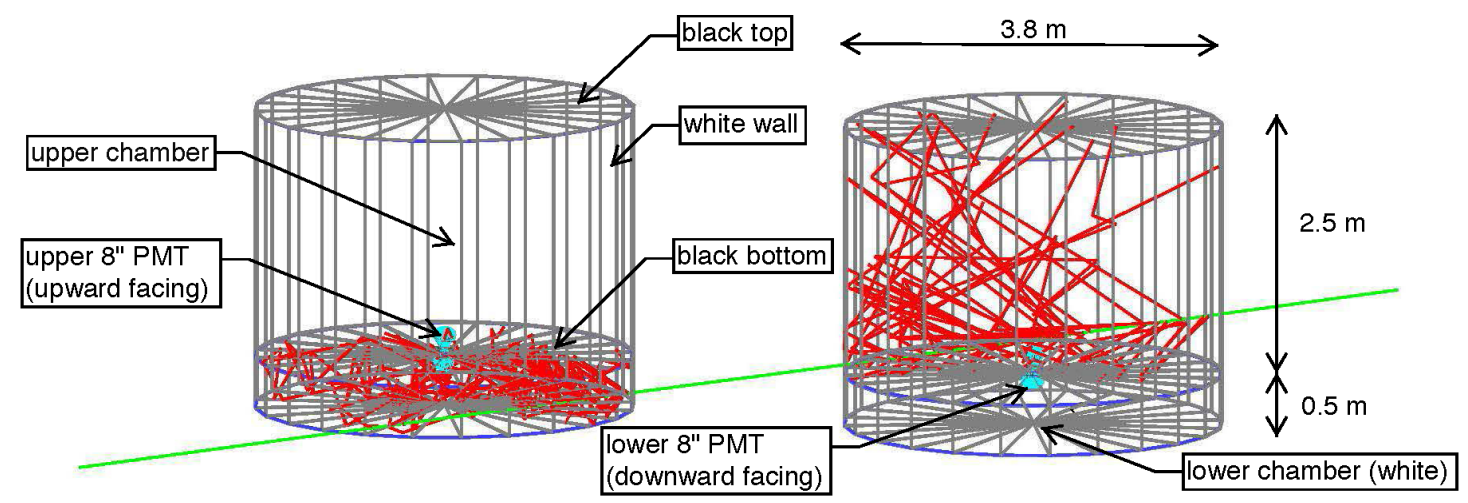

Figure 2: Cylindrical Double Layered WCD designs comprising an upper chamber $\left(\pi \times 1.91^{2} \times 2.5 \mathrm{~m}^{3}\right)$ with white walls and black bases (top and bottom) and an entirely white lower chamber $\left(\pi \times 1.91^{2} \times 0.5 \mathrm{~m}^{3}\right)$. The upper chamber comprises an 8" PMT facing upwards, and the lower chamber comprises an 8" PMT facing downwards. A Muon (green) passes through both units and produces photons (red). The number of photons has been limited here for illustration purposes.

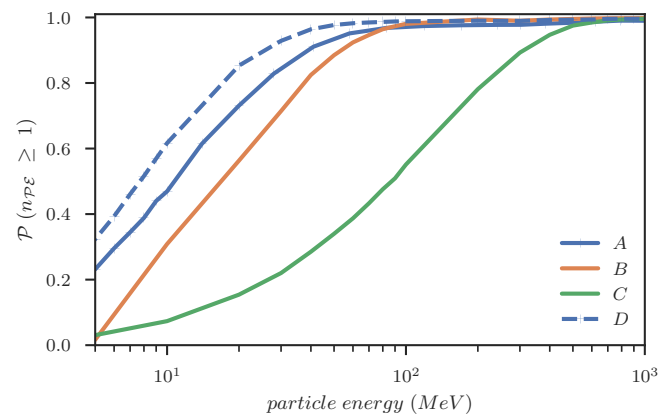

(a)

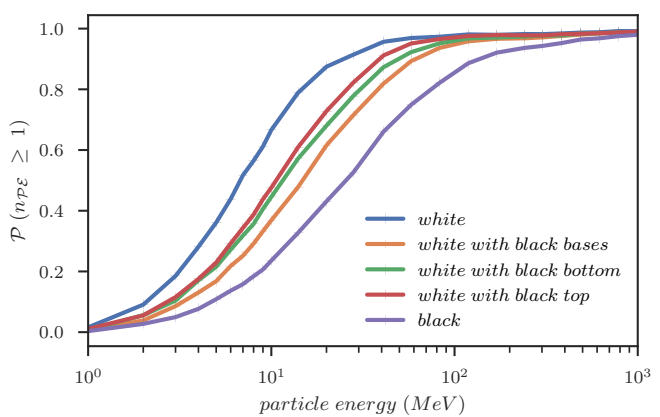

(b)

Figure 3: (a) Injection of vertical $5 \mathrm{MeV}$ to $1 \mathrm{GeV} \gamma$-rays across the top surface of different WCD designs. Here we show a comparison between the upper chamber of a [A] white cylindrical double-layered WCD unit $\left(\pi \times 1.91^{2} \times 2.5 \mathrm{~m}^{3}\right)$ with a black top and an 8" PMT, a [B] HAWC - like design $\left(\pi \times 3.65^{2} \times 4 \mathrm{~m}^{3}\right)$ with black walls, a central 10" PMT and 3x8" PMTs', a [C] LHAASO - like black unit $\left(5 \times 5 \times 4.5 \mathrm{~m}^{3}\right)$ with an open top and an 8" PMT and a [D] white cylindrical double-layered WCD unit $\left(\pi \times 1.71^{2} \times 3 \mathrm{~m}^{3}\right)$ with a black top and an 8" PMT. (b) Response to injection of vertical $1 \mathrm{MeV}$ to $1 \mathrm{GeV} \gamma$-rays across the top of the upper chamber of double-layered WCD unit $\left(\pi \times 1.91^{2} \times 2.5 \mathrm{~m}^{3}\right)$ with vertical $1 \mathrm{MeV}$ to $1 \mathrm{GeV} \gamma$-rays with different materials.

light is detected first. The lower chamber is a similar light-tight chamber but, to ensure we collect all the energy deposited in the chamber for muon identification, it is composed of highly reflective material and a centrally located 8" PMT facing downwards.

\section{Particle Detection Efficiency and Energy Resolution}

The particle detection efficiency of the DLWCD is optimised by leveraging the aspect ratio and the material (reflectivity) selection. To maximise the probability of cascade production, both 


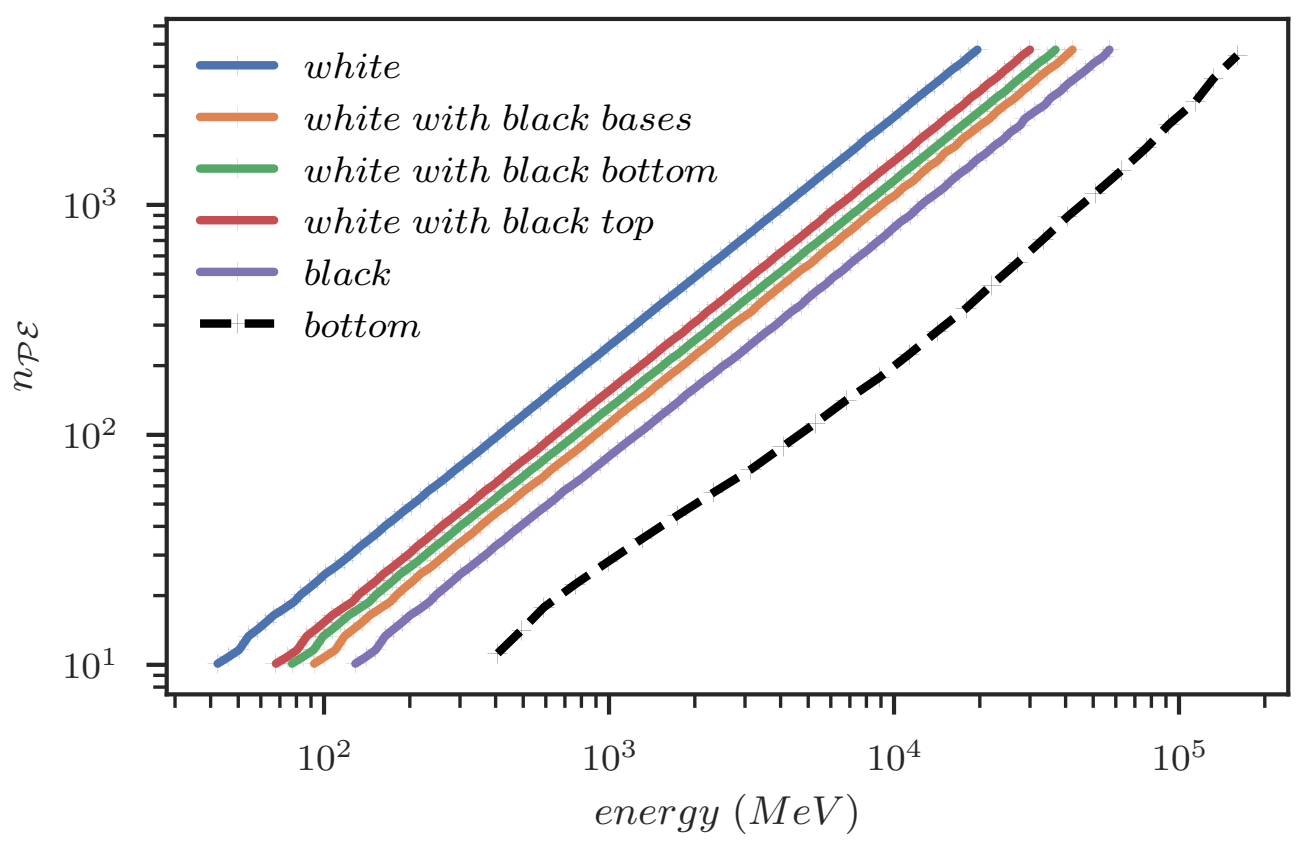

Figure 4: The number of p.e's at different electromagnetic energy for upper chambers with different materials. The dashed black line shows the corresponding value for the lower chamber.

LHAASO and HAWC have a water depth $>10 \times x_{0}$, where $x_{0}$ is the radiation length of high energy $\gamma^{\prime} s$ in water $(\sim 46 \mathrm{~cm})$. However, both design also uses a material with low reflectivity (black) like Polypropylene. The upper chamber of the proposed DLWCD is shallow and narrower than these designs. The chamber also comprises reflective walls, namely, Tyvek used by the Pierre Auger Observatory [15] with a combination of black bases.

To compare the detection efficiency of the DLWCD, vertical $\gamma$ 's were injected across the top of several design choices varying in aspect-ratio and material reflectivity (see Fig. 3). The DLWCD design, with white walls and a black top, has improved particle detection efficiency over both HAWC and LHAASO - like designs. A deeper chamber would ensure cascade production and subsequent detection of Cherenkov photons at $\sim 100 \mathrm{MeV} \gamma s$, while a narrower chamber with reflective walls improves sensitivity to lower energy $\gamma s$. Reflective walls improve particle detection efficiency over non-reflective walls.

Additionally, high energy $\gamma$ 's close to the shower core can result in the saturation of the upper PMT. To mitigate this, since these particles can also punch through into the lower chamber, the lower PMT can extend the dynamic range (see Fig. 4).

\section{Angular Resolution}

In order to compare the angular resolution of the DLWCD (upper $-\pi \times 1.91^{2} \times 2.5 \mathrm{~m}^{3}$ ) of different material combinations, we simulate vertical $\gamma$ initiated showers at the centre of the array shown in Fig. 1. The angular resolution is then computed in several stages.

First, after requiring a minimum of 10 unit hits, a time difference of arrival of the shower hit first arrival times for each unit are used to compute the shower direction and time. Limiting the 


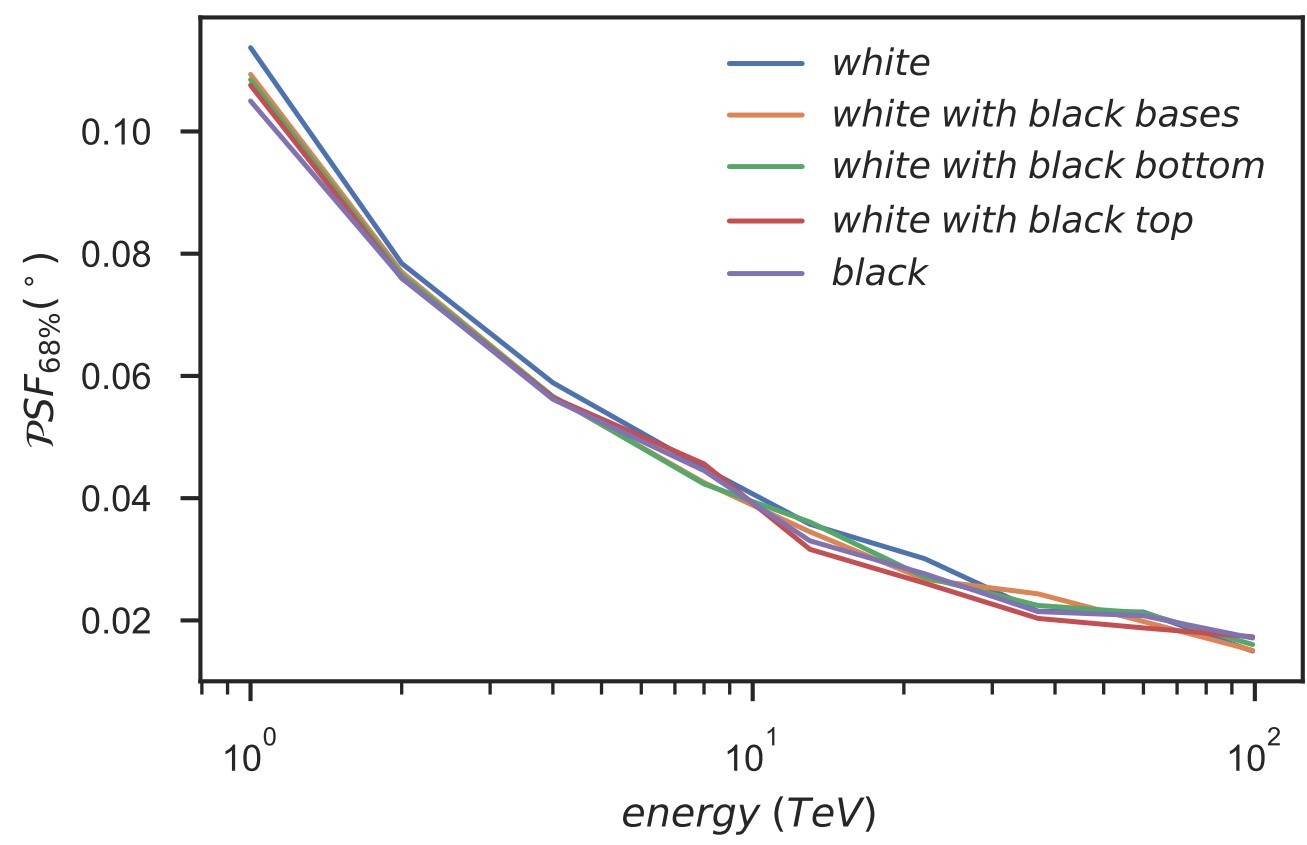

Figure 5: Angular resolution for $1-100 \mathrm{TeV}$ vertical $\gamma$-ray's simulated with shower core at the center of an array of double-layered WCD's (upper $-\pi \times 1.91^{2} \times 2.5 \mathrm{~m}^{3}$ ) with $\sim 80 \%$ fill factor varying the material properties.

shower hits to $10 \%$ of the units hit with the largest charge limits the computational burden. A Landau fit to the arrival times as a function of distance to the shower core and charge is used to obtain mean and width parameters.

Given the Landau fit parameters, a 3-parameter likelihood fit (MINUIT [16]) is implemented to obtain the shower direction. The angular resolution is the $68 \%$ containment of such showers (see Fig. 5).

We find that, as expected, as most of the first photons are the direct Cherenkov light, there is no or limited impact of the material combination on the angular resolution of the showers.

\section{Gamma Hadron Separation}

To evaluate the $\gamma$-hadron separation power, a Template-based maximum log-likelihood method comprising charge in the two chambers is implemented to discriminate between $\gamma$-ray and hadron induced air showers for an ensemble of $\gamma$-ray and proton-induced vertical showers of 1 to $100 \mathrm{TeV}$ energy with the shower core located at the centre of the array. First, $\gamma$-ray and proton initiated showers are split into $70-30 \%$ training and test sets, respectively, with an exclusion region of $40 \mathrm{~m}$. The exclusion region is defined to account for the high transverse momentum of $\mu^{ \pm}$and punch-through of $\gamma \& e^{ \pm}$close to the shower core. The training set is then used to generate separate templates of charge in the upper and lower chambers for $\mu^{ \pm}$and $e^{ \pm}, \& \gamma$ 's. The test set is then used to identify the likelihood of a $\mu^{ \pm}$on a tank-by-tank basis.

Once $\mu^{ \pm}$are tagged, the number of such particles is counted on an event-by-event basis for both $\gamma$ and hadron initiated showers for a similar number of tanks hit. The $\gamma$ and $p^{+}$identification 


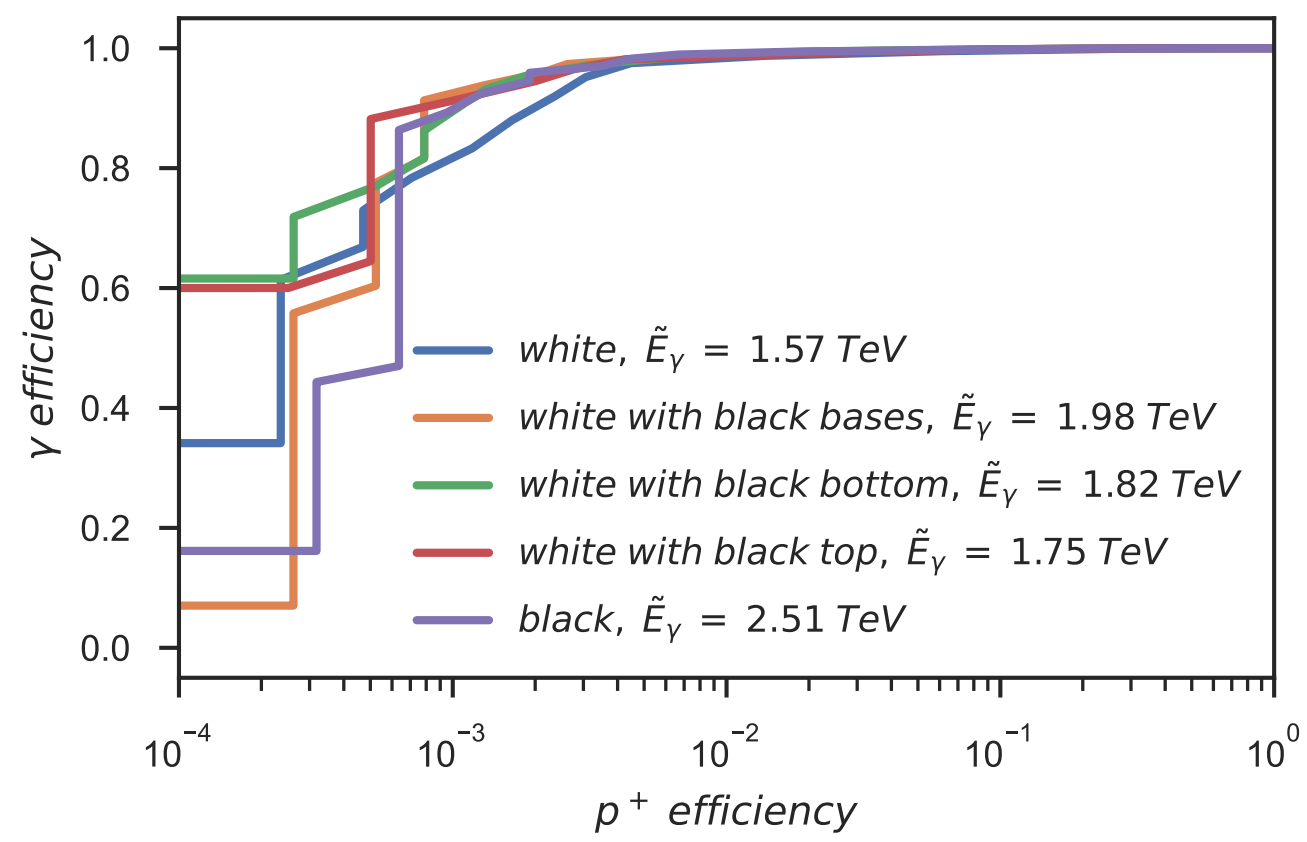

Figure 6: Gamma - Hadron separation efficiency for an array of double-layered WCD's (upper $-\pi \times$ $1.91^{2} \times 2.5 \mathrm{~m}^{3}$ ) with $\sim 80 \%$ fill factor varying material reflectivity and an exclusion region of $40 \mathrm{~m}$ for $547 \leq$ nhits $<1280$.

efficiency is shown in Fig. 6 for different material combinations and $547 \leq$ nhits $<1280$. Tanks with a combination of white material represent showers with a lower median $\gamma$-ray energy. As expected while there is no significant difference in the $\gamma$ - hadron separation power with different material combinations, with the combination of reflective material lower energy threshold can be achieved due to an increased particle detection efficiency.

\section{Conclusion}

The Southern Wide-field-of-view Gamma-ray Observatory (SWGO) will use the well-established and cost-effective technique of detecting Cherenkov light produced in water-filled detection units for $\mathrm{TeV}$ gamma-ray astronomy. Several detector technologies such as units with multiple photo-sensors and an option to deploy detector units in a lake are currently under investigation. The double-layered WCD leverages material and aspect ratio to enhance sensitivity, achieve excellent angular resolution and gamma hadron separation.

\section{Acknowledgements}

The SWGO Collaboration acknowledges the support from the agencies and organizations listed here: https://www . swgo .org/SWGOWiki/doku. php?id=acknowledgements. 


\section{References}

[1] A. U. Abeysekara et al. (HAWC Collaboration), Astrophys. J. 843, 39 (2017).

[2] G. D. Sciascio et al. (LHAASO Collaboration), Nucl. Part. Phys. Proc. 279 - 281, 166-173 (2016).

[3] Z. Cao, F.A. Aharonian, Q. An et al. Ultrahigh-energy photons up to 1.4 petaelectronvolts from $12 \gamma$-ray Galactic sources. Nature 594, 33-36 (2021). https://doi.org/10.1038/s41586021-03498-z

[4] A. Albert et al. (SGSO Alliance), arXiv:1902.08429 (2019).

[5] J. Hinton (SWGO Collaboration), PoS ICRC2021 (2021) 023. https://pos.sissa.it/ $395 / 023 / p d f$

[6] R. Conceicao (SWGO Collaboration), PoS ICRC2021 (2021) 707. https://pos.sissa. it $/ 395 / 707 / p d f$

[7] H. Goksu (SWGO Collaboration), PoS ICRC2021 (2021) 708. https://pos.sissa.it/ $395 / 708 / p d f$

[8] H. Schoorlemmer et al., Eur. Phys. J. C 79, 427 (2019). https: //doi . org/10 . 1140/epjc/ s10052-019-6942-x

[9] S. Agostinelli et al., Nucl. Instrum. Methods Phys. Res. A 506 205-303 (2003).

[10] Heck, D., et al., CORSIKA: a Monte Carlo code to simulate extensive air showers. (FZKA 6019)(Karlsruhe: Forschungszentrum Karlsruhe) (1998).

[11] S. Ostapchenko, Phys. Rev. D 83, 014018 (2011). https: //doi .org/10 . 1103/PhysRevD. 83.014018

[12] S.A.Bass et al. Prog.Part.Nucl.Phys. 41 (1998) 225

[13] M.Bleicher et al. J.Phys. G25 (1999) 1859

[14] Nelson, W. \& Namito, Yoshihito. (1990). The EGS4 Code System: Solution of gamma-ray and electron transport problems.

[15] I. Allekotte et al. (Pierre Auger Collaboration), Nuclear Instruments and Methods in Physics Research A 586, 409-420 (2008).

[16] F. James, MINUIT Function Minimization and Error Analysis: Reference Manual Version, 94.1, CERN-D-506 (2017) 


\section{Full Authors List: SWGO Collaboration}

P. Abreu ${ }^{1}$, A. Albert ${ }^{2}$, E. O. Angüner ${ }^{3}$, C. Arcaro $^{4}$, L.H. Arnaldi ${ }^{5}$, J.C. Arteaga-Velázquez ${ }^{6}$, P. Assis ${ }^{1}$, A. Bakalová ${ }^{7}$, U. Barres de Almeida ${ }^{8}$, I. Batković ${ }^{4}$, J. Bellido ${ }^{9}$, E. Belmont-Moreno ${ }^{10}$, F. Bisconti ${ }^{11}$, A. Blanco ${ }^{1}$, M. Bohacova ${ }^{7}$, E. Bottacini ${ }^{4}$, T. Bretz ${ }^{12}$, C. Brisbois ${ }^{13}$, P. Brogueira ${ }^{1}$, A. M. Brown ${ }^{14}$, T. Bulik ${ }^{15}$, K. S. Caballero Mora ${ }^{16}$, S. M. Campos ${ }^{17}$ A. Chiavassa ${ }^{11}$, L. Chytka ${ }^{7}$, R. Conceição ${ }^{1}$, G. Consolati ${ }^{18}$, J. Cotzomi Paleta ${ }^{19}$, S. Dasso ${ }^{20}$, A. De Angelis ${ }^{4}$, C.R. De Bom ${ }^{8}$, E. de la Fuente ${ }^{21}$, V. de Souza ${ }^{22}$, D. Depaoli ${ }^{11}$, G. Di Sciascio ${ }^{23}$, C. O. Dib ${ }^{24}$, D. Dorner ${ }^{25}$, M. Doro ${ }^{4}$, M. Du Vernois ${ }^{26}$, T. $\operatorname{Ergin}^{27}$, K. L. Fan ${ }^{13}$, N. Fraija ${ }^{8}$, S. Funk ${ }^{28}$, J. I. García ${ }^{17}$, J. A. García-González ${ }^{29}$, S. T. García Roca ${ }^{9}$, G. Giacinti ${ }^{30}$, H. Goksu ${ }^{30}$, B. S. González ${ }^{1}$, F. Guarino ${ }^{31}$, A. Guillén ${ }^{32}$,

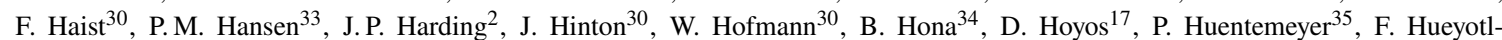
Zahuantitla $^{16}$ A. Insolia ${ }^{36}$, P. Janecek ${ }^{7}$, V. Joshi ${ }^{28}$, B. Khelifi ${ }^{37}$, S. Kunwar ${ }^{30}$, G. La Mura ${ }^{1}$, J. Lapington ${ }^{38}$, M. R. Laspiur ${ }^{17}$, F. Leit1 ${ }^{28}$, F. Longo ${ }^{39}$, L. Lopes ${ }^{1}$, R. Lopez-Coto ${ }^{4}$, D. Mandat ${ }^{7}$, A. G. Mariazzi ${ }^{33}$, M. Mariotti ${ }^{4}$, A. Marques Moraes ${ }^{8}$, J. MartínezCastro $^{40}$, H. Martínez-Huerta ${ }^{41}$, S. May ${ }^{42}$, D. G. Melo ${ }^{43}$, L. F. Mendes ${ }^{1}$, L. M. Mendes ${ }^{1}$, T. Mineeva ${ }^{24}$, A. Mitchell ${ }^{44}$, S. Mohan ${ }^{35}$, O. G. Morales Olivares ${ }^{16}$, E. Moreno-Barbosa ${ }^{19}$, L. Nellen ${ }^{45}$, V. Novotny ${ }^{7}$, L. Olivera-Nieto ${ }^{30}$, E. Orlando ${ }^{39}$, M. Pech $^{7}$, A. Pichel $^{20}$, M. Pimenta ${ }^{1}$, M. Portes de Albuquerque ${ }^{8}$, E. Prandini ${ }^{4}$, M. S. Rado Cuchills ${ }^{9}$, A. Reisenegger ${ }^{46}$, B. Reville ${ }^{30}$, C. D. Rho ${ }^{47}$, A. C. Rovero ${ }^{20}$ E. Ruiz-Velasco ${ }^{30}$, G. A. Salazar ${ }^{17}$, A. Sandoval ${ }^{10}$, M. Santander ${ }^{42}$, H. Schoorlemmer ${ }^{30}$, F. Schüssler ${ }^{48}$, V. H. Serrano ${ }^{17}$, R. C. Shellard ${ }^{8}$, A. Sinha ${ }^{49}$, A. J. Smith ${ }^{13}$, P. Surajbali ${ }^{30}$, B. Tomé ${ }^{1}$, I. Torres Aguilar ${ }^{50}$, C. van Eldik ${ }^{28}$, I. D. Vergara-Quispe ${ }^{33}$, A. Viana ${ }^{22}$, J. Vícha ${ }^{7}$, C.F. Vigorito ${ }^{11}$, X. Wang ${ }^{35}$, F. Werner ${ }^{30}$, R. White ${ }^{30}$, M. A. Zamalloa Jara ${ }^{9}$

${ }^{1}$ Laboratório de Instrumentação e Física Experimental de Partículas (LIP), Av. Prof. Gama Pinto 2, 1649-003 Lisboa, Portugal

2 Physics Division, Los Alamos National Laboratory, P.O. Box 1663, Los Alamos, NM 87545, United States

${ }^{3}$ Aix Marseille Univ, CNRS/IN2P3, CPPM, 163 avenue de Luminy - Case 902, 13288 Marseille cedex 09, France

${ }^{4}$ University of Padova, Department of Physics and Astronomy \& INFN Padova, Via Marzolo 8 - 35131 Padova, Italy

${ }^{5}$ Centro Atómico Bariloche, Comisión Nacional de Energía Atómica, S. C. de Bariloche (8400), RN, Argentina

${ }^{6}$ Universidad Michoacana de San Nicolás de Hidalgo, Calle de Santiago Tapia 403, Centro, 58000 Morelia, Mich., México

${ }^{7}$ FZU, Institute of Physics of the Czech Academy of Sciences, Na Slovance 1999/2, 18200 Praha 8, Czech Republic

${ }^{8}$ Centro Brasileiro de Pesquisas Físicas, R. Dr. Xavier Sigaud, 150 - Rio de Janeiro - RJ, 22290-180, Brazil

${ }^{9}$ Academic Department of Physics - Faculty of Sciences - Universidad Nacional de San Antonio Abad del Cusco (UNSAAC), Av. de la Cultura, 733, Pabellón C-358, Cusco, Peru

${ }^{10}$ Instituto de Física, Universidad Nacional Autónoma de México, Sendero Bicipuma, C.U., Coyoacán, 04510 Ciudad de México, CDMX, México

${ }^{11}$ Dipartimento di Fisica, Università degli Studi di Torino, Via Pietro Giuria 1, 10125, Torino, Italy

${ }^{12}$ RWTH Aachen University, Physics Institute 3, Otto-Blumenthal-Straße, 52074 Aachen, Germany

${ }^{13}$ University of Maryland, College Park, MD 20742, United States

${ }^{14}$ Durham University, Stockton Road, Durham, DH1 3LE, United Kingdom

${ }^{15}$ Astronomical Observatory, University of Warsaw, Aleje Ujazdowskie 4, 00478 Warsaw, Poland

${ }^{16}$ Facultad de Ciencias en Física y Matemáticas UNACH, Boulevard Belisario Domínguez, Km. 1081, Sin Número, Terán, Tuxtla Gutiérrez, Chiapas, México

${ }^{17}$ Facultad de Ciencias Exactas, Universidad Nacional de Salta, Avda. Bolivia No 5150, (4400) Salta Capital, Argentina

${ }^{18}$ Department of Aerospace Science and Technology, Politecnico di Milano, Via Privata Giuseppe La Masa, 34, 20156 Milano MI, Italy

${ }^{19}$ Facultad de Ciencias Físico Matemáticas, Benemérita Universidad Autónoma de Puebla, C.P. 72592, México

${ }^{20}$ Instituto de Astronomia y Fisica del Espacio (IAFE, CONICET-UBA), Casilla de Correo 67 - Suc. 28 (C1428ZAA), Ciudad Autónoma de Buenos Aires, Argentina

${ }^{21}$ Universidad de Guadalajara, Blvd. Gral. Marcelino García Barragán 1421, Olímpica, 44430 Guadalajara, Jal., México

22 Instituto de Física de São Carlos, Universidade de São Paulo, Avenida Trabalhador São-carlense, no 400, Parque Arnold Schimidt CEP 13566-590, São Carlos - São Paulo - Brasil

${ }^{23}$ INFN - Roma Tor Vergata and INAF-IAPS, Via del Fosso del Cavaliere, 100, 00133 Roma RM, Italy

${ }^{24}$ Dept. of Physics and CCTVal, Universidad Tecnica Federico Santa Maria, Avenida España 1680, Valparaíso, Chile

${ }^{25}$ Universität Würzburg, Institut für Theoretische Physik und Astrophysik, Emil-Fischer-Str. 31, 97074 Würzburg, Germany

${ }^{26}$ Department of Physics, and the Wisconsin IceCube Particle Astrophysics Center (WIPAC), University of Wisconsin, 222 West Washington Ave., Suite 500, Madison, WI 53703, United States

27 TUBITAK Space Technologies Research Institute, ODTU Campus, 06800, Ankara, Turkey

${ }^{28}$ Friedrich-Alexander-Universität Erlangen-Nürnberg, Erlangen Centre for Astroparticle Physics, Erwin-Rommel-Str. 1, D 91058 Erlangen, Germany

${ }^{29}$ Tecnologico de Monterrey, Escuela de Ingeniería y Ciencias, Ave. Eugenio Garza Sada 2501, Monterrey, N.L., 64849, México

${ }^{30}$ Max-Planck-Institut für Kernphysik, P.O. Box 103980, D 69029 Heidelberg, Germany

${ }^{31}$ Università di Napoli "Federico II", Dipartimento di Fisica "Ettore Pancini", and INFN Napoli, Complesso Universitario di Monte Sant'Angelo - Via Cinthia, 21 - 80126 - Napoli, Italy

${ }^{32}$ University of Granada, Campus Universitario de Cartuja, Calle Prof. Vicente Callao, 3, 18011 Granada, Spain 
33 IFLP, Universidad Nacional de La Plata and CONICET, Diagonal 113, Casco Urbano, B1900 La Plata, Provincia de Buenos Aires, Argentina

19734 University of Utah, 201 Presidents' Cir, Salt Lake City, UT 84112, United States

35 Michigan Technological University, 1400 Townsend Drive, Houghton, MI 49931, United States

36 Dipartimento di Fisica e Astronomia "E. Majorana", Catania University and INFN, Catania, Italy

${ }^{38}$ University of Leicester, University Road, Leicester LE1 7RH, United Kingdom

${ }^{39}$ Department of Physics, University of Trieste and INFN Trieste, via Valerio 2, I-34127, Trieste, Italy

${ }^{40}$ Centro de Investigación en Computación, Instituto Politécnico Nacional, Av. Juan de Dios Bátiz S/N, Nueva Industrial Vallejo, Gustavo A. Madero, 07738 Ciudad de México, CDMX, México

${ }^{41}$ Department of Physics and Mathematics, Universidad de Monterrey, Av. Morones Prieto 4500, San Pedro Garza García 66238, N.L., México

42 Department of Physics and Astronomy, University of Alabama, Gallalee Hall, Tuscaloosa, AL 35401, United States

43 Instituto de Tecnologías en Detección y Astropartículas (CNEA-CONICET-UNSAM), Av. Gral Paz 1499 - San Martín - Pcia. de Buenos Aires, Argentina

44 Department of Physics, ETH Zurich, CH-8093 Zurich, Switzerland

45 Instituto de Ciencias Nucleares, Universidad Nacional Autónoma de México (ICN-UNAM), Cto. Exterior S/N, C.U., Coyoacán, 04510 Ciudad de México, CDMX, México

46 Departamento de Física, Facultad de Ciencias Básicas, Universidad Metropolitana de Ciencias de la Educación, Av. José Pedro Alessandri 774, Ñuñoa, Santiago, Chile

47 Department of Physics, University of Seoul, 163 Seoulsiripdaero, Dongdaemun-gu, Seoul 02504, Republic of Korea

${ }^{48}$ Institut de recherche sur les lois fondamentales de l'Univers (IRFU), CEA, Université Paris-Saclay, F-91191 Gif-sur-Yvette, France

${ }^{49}$ Laboratoire Univers et Particules de Montpellier, CNRS, Université de Montpelleir, F-34090 Montpellier, France

${ }^{50}$ Instituto Nacional de Astrofísica, Óptica y Electrónica (INAOE), Luis Enrique Erro 1, Puebla, México 